\title{
Fixed Point of Generalized Weak Contraction in $b$-Metric Spaces
}

\author{
Maryam Iqbal $\mathbb{D},{ }^{1}$ Afshan Batool $\mathbb{D},{ }^{1}$ Ozgur Ege $\mathbb{D}^{,},{ }^{2}$ and Manuel de la Sen $\mathbb{D}^{3}$ \\ ${ }^{1}$ Department of Mathematical Sciences, Fatima Jinnah Women University, Rawalpindi, Pakistan \\ ${ }^{2}$ Department of Mathematics, Faculty of Science, Ege University, Bornova, 35100 Izmir, Turkey \\ ${ }^{3}$ Institute of Research and Development of Processes, University of the Basque Country, 48940 Leioa, Spain
}

Correspondence should be addressed to Ozgur Ege; ozgur.ege@ege.edu.tr

Received 5 April 2021; Accepted 4 June 2021; Published 16 June 2021

Academic Editor: Santosh Kumar

Copyright (c) 2021 Maryam Iqbal et al. This is an open access article distributed under the Creative Commons Attribution License, which permits unrestricted use, distribution, and reproduction in any medium, provided the original work is properly cited.

In this manuscript, a class of generalized $(\psi, \alpha, \beta)$-weak contraction is introduced and some fixed point theorems in the framework of $b$-metric space are proved. The result presented in this paper generalizes some of the earlier results in the existing literature. Further, some examples and an application are provided to illustrate our main result.

\section{Introduction and Preliminaries}

Fixed point theory plays a vital role in the development of nonlinear functional analysis. It has been used in various branches of engineering and sciences. Banach contraction principle is one of the most important results in fixed point theory introduced by great Polish mathematician Stefan Banach [1]. The concept of $b$-metric space or metric-type space was first introduced by Czerwik [2]. He provided a property which is weaker than the triangular inequality. The basic idea of $b$-metric was commenced by Bourbaki [3] and Bakhtin [4]. Later on, Khamsi and Hussain [5] reintroduced such spaces under the name of metric-type spaces for some results of fixed and common fixed points in the setting of $b$-metric spaces. Since then, several authors proved fixed point results of single valued and multivalued operators in $b$-metric space and its different type generalizations, we refer [6-22]. Every one of these applications captivated us to present the idea of $b$-metric space.

Definition 1. (see [23]). Let $X$ be a nonempty set. A function $d: X \times X \longrightarrow 0, \infty$ is said to be a $b$-metric if it satisfies the following conditions:

$$
\begin{aligned}
& \text { (1) } 0 \leq d(x, y) \text { and } d(x, y)=0 \text { if and only if } x=y \\
& \text { (2) } d(x, y)=d(y, x) \\
& \text { (3) } d(x, z) \leq s[d(x, y)+d(y, z)] \text { for some } s \geq 1
\end{aligned}
$$

for all $x, y, z \in X$. The pair $(X, d)$ is called a $b$-metric with coefficient $s$.

Here, we observe that every metric space is a $b$-metric with $s=1$. Conditions (1) and (2) of Definition 1 are similar to metric space but it is important how to use (3) effectively. An example is given to expound the concept of a third condition.

Example 1. Let $X=\mathbb{R}$. We define a mapping $d: X \times X \longrightarrow$ $\mathbb{R}^{+}$such that

$$
d(x, y)=(x-y)^{2}, \forall x, y \in X .
$$

The first two conditions of Definition 1 are clearly shown. The solution of third condition is as follows:

$$
\begin{aligned}
d(x, z)= & (x-z)^{2}=x^{2}+z^{2}-2 x z \leq x^{2}+z^{2}-2 x z \\
& +x^{2}+z^{2}+4 y^{2}-4 x y-2 .
\end{aligned}
$$

Since

$$
x^{2}+z^{2}+4 y^{2}>2 x(2 y+z), x^{2}+z^{2}+4 y^{2}-2 x(2 y+z)>0,
$$


we have

$$
\begin{aligned}
d(x, z) & \leq x^{2}+z^{2}-2 x z+x^{2}+z^{2}+4 y^{2}-4 x y-2 \\
& =2 x^{2}+4 y^{2}+2 z^{2}-4 x y-4 x z \\
& =2\left[x^{2}+2 y^{2}+z^{2}-2 x y-2 x z\right] \\
& =2\left[x^{2}+y^{2}-2 x y+y^{2}+z^{2}-2 x z\right] \\
& =2\left[(x-y)^{2}+(y-z)^{2}\right] \\
& =2[d(x, y)+d(y, z)] .
\end{aligned}
$$

Then, we obtain

$$
d(x, z) \leq 2[d(x, y)+d(y, z)] .
$$

So the value of coefficient is $s=2$.

In this section, the concept of generalized $(\psi, \alpha, \beta)$-weak contraction for metric space is provided with some basic notions and results. In 1997, Alber and Guerre-Delabriere [24] suggested a generalization of Banach contraction mapping by introducing the concept of $\phi$-weak contraction in Hilbert space. In 2008, Dutta and Choudhury [25] gave a generalization of weakly contractive mapping by defining $(\psi, \phi)$-weak contraction in complete metric spaces.

Definition 2. (see [26]). Let $\Psi$ denote the class of function $\psi:[0, \infty) \longrightarrow[0, \infty)$ which satisfies the following conditions:

(1) $\psi$ is continuous and nondecreasing

$$
\psi(t)=0 \Longleftrightarrow t=0 .
$$

Definition 3. (see [26]). A self-map $P$ is said to be a weakly contractive map if there exists a function $\phi:[0,+\infty) \longrightarrow[0$, $+\infty)$ such that $\phi$ is continuous, nondecreasing, and $\phi(t)=0$ $\Longleftrightarrow t=0$ and satisfying

$$
d(P x, P y) \leq d(x, y)-\phi(d(x, y)), \forall x, y \in X .
$$

Theorem 4. (see [26]). Let $(X, d)$ be a complete metric space and $P$ be a weakly contractive self-map on $X$. Then, $P$ has a unique fixed point in $X$.

Definition 5. (see [25]). A self-map $P$ is said to be $(\psi, \phi)$-weak contraction, if for each $x, y \in X$,

$$
\psi(d(P x, P y)) \leq \psi(d(x, y))-\phi(d(x, y)),
$$

where $\psi, \phi:[0, \infty] \longrightarrow[0, \infty]$ are both continuous and monotone nondecreasing functions with $\psi(t)=0=\phi(t)$ $\Longleftrightarrow t=0$.

Theorem 6. (see [25]). Let $(X, d)$ be a complete metric space and a self-map $P$ be a $(\psi, \phi)$-weak contraction. Then, $P$ has a unique fixed point.

Definition 7. (see [27]) Two self-maps $P$ and $Q$ are said to be generalized $\phi$-weakly contractive map if there exists a func- tion $\phi:[0,+\infty] \longrightarrow[0,+\infty]$ such that $\phi$ is continuous, nondecreasing, and $\phi(t)=0 \Longleftrightarrow t=0$ and satisfying

$$
d(P x, Q y) \leq M(x, y)-\phi(M(x, y))
$$

where

$M(x, y)=\max \left\{d(x, y), d(x, P x), d(y, Q y), \frac{[d(y, P x)+d(x, Q y)]}{2}\right\}, \forall x, y \in X$.

Theorem 8. (see [27]). Let $(X, d)$ be a complete metric space and $T$ and $R$ are generalized $\phi$-weakly contractive self-maps on $X$. Then, $P$ and $Q$ have a unique common fixed point in $X$.

Definition 9. (see [28]). Two self-maps $P$ and $Q$ are said to be generalized $(\phi, \psi)$-weakly contractive maps if they satisfy

$$
\psi(P x, Q y) \leq M(x, y)-\phi(M(x, y))
$$

$\forall x, y \in X$, where $\phi:[0,+\infty) \longrightarrow[0,+\infty)$ such that $\psi$ is continuous, nondecreasing, and $\psi(t)=0 \Longleftrightarrow t=0, \phi:[0,+$ $\infty] \longrightarrow[0,+\infty)$ such that $\phi$ is a lower semicontinuous function, $\phi(t)=0 \Longleftrightarrow t=0$ and

$M(x, y)=\max \left\{d(x, y), d(x, P x), d(y, Q y), \frac{[d(y, P x)+d(x, Q y)]}{2}\right\}, \forall x, y \in X$.

Theorem 10. (see [28]). Let $(X, d)$ be a complete metric space and $P$ and $Q$ are generalized $(\phi, \psi)$-weakly contractive selfmaps on $X$. Then, $P$ and $Q$ have a unique common fixed point in $X$.

Definition 11. (see [29]). Let $(X, \preccurlyeq)$ be a partially ordered set. Two mappings $P, Q: X \longrightarrow X$ are said to be weakly increasing if $P x \leqslant Q T x$ and $Q x \leqslant P Q x$ for all $x \in X$.

Remark 12. (see [29]). Note that two weakly increasing mappings need not be nondecreasing.

Definition 13. (see [30]). Let $(X, d)$ be a metric space and $P, Q: X \longrightarrow X$ are given two self-mappings on $X$. The pair $(P, Q)$ is said to be compatible if $\lim _{n \longrightarrow \infty} d\left(P Q x_{n}, Q P x_{n}\right)$ $=0$, whenever $x_{n}$ is a sequence in $X$ such that

$$
\lim _{n \longrightarrow \infty} P x_{n}=\lim _{n \longrightarrow \infty} Q x_{n}=t \text {, for some } t \in X .
$$

Definition 14. (see [1]). Let $(X, \preccurlyeq)$ be a partially ordered set and let $(X, d)$ be a $b$-metric space with coefficient $s \geq 1$. Three maps $T, R$, and $S$ are said to be a generalized $(\psi, \alpha, \beta)$-weak contraction if for each $x, y \in X$,

$$
\psi(d(P x, Q y)) \leq \alpha((d(S x, S y)) \beta(d(S x, S y)),
$$

where $\psi \in \Psi$ and $\beta:[0, \infty) \longrightarrow[0, \infty)$ is a continuous function with condition 


$$
0<\beta(t)<\psi(t), \forall t>0
$$

Theorem 15. (see [1]). Let $(X, \preccurlyeq)$ be a partially ordered set and assume that there exists a metric function $d$ in $X$ such that $(X, d)$ is a complete metric space. Let $P, Q, S: X \longrightarrow X$ are generalized $(\psi, \alpha, \beta)$-weak contraction mappings satisfying the following properties:

$$
P X \subseteq S X,
$$

(1) $P, Q$, and $S$ are continuous

(2) The pairs $(P, S)$ and $(Q, S)$ are compatible

(3) $P$ and $Q$ weakly increasing with respect to $S$

(4) Sx and Sy are comparable

(5) $\forall(x, y) \in X \times X$, there exists $u \in X$ such that $P x \preccurlyeq P u$ and $P y \leqslant P u$

Then, $P, Q$, and $S$ have a unique common fixed point $z \in X$.

The next section includes the concept of generalized $(\psi, \alpha, \beta)$-weak contraction for $b$-metric space and theorem related to it.

\section{Generalized $(\psi, \alpha, \beta)$-Weak Contractions}

Definition 16 . Let $(X, \preccurlyeq)$ be a partially ordered set and let $(X, d)$ be a $b$-metric space with coefficient $s \geq 1$. Three maps $P, Q$, and $S$ are said to be a generalized $(\psi, \alpha, \beta)$-weak contraction if for each $x, y \in X$ and $b \in(0,1)$.

$$
b^{s} \psi(d(P x, Q y)) \leq \alpha((d(S x, S y)) \beta(d(S x, S y)), \forall x \geq y,
$$

where $\alpha \in F, \psi \in \Psi$ and $\beta:[0, \infty) \longrightarrow[0, \infty)$ is a continuous function with the condition

$$
0<\beta(t)<\psi(t), \forall t>0
$$

Theorem 17. Let $(X, \preccurlyeq)$ be a partially ordered set and assume that there exist $a b$-metric function $d$ in $X$ such that $(X, d)$ is complete b-metric space. Let $T, R, S: X \longrightarrow X$ are generalized $(\psi, \alpha, \beta)$-weak contraction mappings satisfying the following properties:

$P X \subseteq S X$,

(1) $P, Q$, and $S$ are continuous

(2) The pair $(P, S)$ and $(Q, S)$ are compatible

(3) $P$ and $Q$ weakly increasing with respect to $S$

(4) Sx and Sy are comparable
(5) $\forall(x, y) \in X \times X$, there exist $u \in X$ such that $P x \leqslant P u$ and $P y \leqslant P u$

Then, $P, Q$, and S have a unique common fixed point $z \in X$.

Proof. The proof is done by using the concept of Banach contraction principle in which a Cauchy sequence is taken in complete $b$-metric space. Every Cauchy sequence is convergent in a complete metric space, and converging point of that sequence is proved to be a fixed point of contraction.

Let us assume that $x_{0} \in X$ be an arbitrary point in $X$. By property (1), there exist $x_{1}, x_{2} \in X$ such that $P x_{0}=S x_{1}$ and $Q x_{1}=S x_{2}$. Continuing this process, sequences $\left\{x_{n}\right\}$ and $\left\{y_{n}\right\}$ can be constructed in $X$, defined as

$$
S x_{2 n+1}=P x_{2 n}=y_{2 n}, S x_{2 n+1}=Q x_{2 n+1}=y_{2 n+1}, \forall n \in \mathbb{N} \text {. }
$$

By using property (4), we obtain

$$
S x_{1}=P x_{0} \preccurlyeq Q x_{1}=S x_{2} .
$$

Similarly,

$$
S x_{2}=P x_{1} \preccurlyeq Q x_{2}=S x_{3}
$$

Continuing this process, we get

$$
S x_{1} \preccurlyeq S x_{2} \preccurlyeq S x_{3} \preccurlyeq S x_{4} \preccurlyeq \cdots \preccurlyeq S x_{2 n+1} \preccurlyeq S x_{2 n+2} \preccurlyeq \cdots
$$

Thus,

$$
y_{0} \preccurlyeq y_{1} \preccurlyeq y_{2} \preccurlyeq \cdots \preccurlyeq y_{2 n} \preccurlyeq y_{2 n+1} \preccurlyeq \cdots .
$$

According to our first supposition, if there exists $n \in \mathbb{N}$ such that $y_{2 n-1}=y_{2 n}$, then from (18),

$$
\begin{aligned}
b^{s} \psi\left(d\left(y_{2 n}, y_{2 n+1}\right)\right) & =b^{s} \psi\left(d\left(P x_{2 n}, Q x_{2 n+1}\right)\right) \\
& \leq b^{s} \alpha\left(d\left(S x_{2 n}, S x_{2 n+1}\right)\right) \beta\left(d\left(S x_{2 n}, S x_{2 n+1}\right)\right) \\
& =b^{s} \alpha\left(d\left(y_{2 n-1}, y_{2 n}\right)\right) \beta\left(d\left(y_{2 n-1}, y_{2 n}\right)\right) \\
& =0,
\end{aligned}
$$

which implies that $y_{2 n+1}=y_{2 n}$. Consequently, $y_{m}=y_{2 n-1}$ for any $m \geq 2 n$. Hence, for every $m \geq 2 n$, we have $S x_{m}=S x_{2 n}$ which implies that $\left\{S x_{n}\right\}$ is a $b$-Cauchy sequence.

According to our second supposition, $y_{n} \neq y_{n+1}$ for any integer $n$. Let $z_{n}=d\left(y_{n}, y_{n+1}\right)$. Now, we have to show that $z_{n} \longrightarrow 0$ as $n \longrightarrow \infty$. Since $S x_{2 n}$ and $S x_{2 n+1}$ are comparable, then from (18), we have

$$
\begin{aligned}
b^{s} \psi\left(d\left(y_{2 n+2}, y_{2 n+1}\right)\right) & =b^{s} \psi\left(d\left(S x_{2 n+3}, S x_{2 n+2}\right)\right) \\
& =b^{s} \psi\left(d\left(P x_{2 n+2}, Q x_{2 n+1}\right)\right) \\
& \leq b^{s} \alpha\left(d\left(S x_{2 n+2}, S x_{2 n+1}\right)\right) \beta\left(d\left(S x_{2 n+2}, S x_{2 n+1}\right)\right) \\
& =b^{s} \alpha\left(d\left(y_{2 n+1}, y_{2 n}\right)\right) \beta\left(d\left(y_{2 n+1}, y_{2 n}\right)\right) .
\end{aligned}
$$


By property (2) of $\psi$ and the fact that $\alpha \in F$, we get

$$
d\left(y_{2 n+2}, y_{2 n+1}\right) \leq d\left(y_{2 n+1}, y_{2 n}\right)
$$

Similarly, we have

$$
d\left(y_{2 n+1}, y_{2 n}\right) \leq d\left(y_{2 n}, y_{2 n-1}\right)
$$

By combining (28) and (29), we obtain

$$
d\left(y_{2 n+2}, y_{2 n+1}\right) \leq d\left(y_{2 n+1}, y_{2 n}\right) \leq d\left(y_{2 n}, y_{2 n-1}\right)
$$

This shows that the sequence $\left\{z_{n}\right\}$ is monotonically decreasing. So there exists $r \geq 0$ such that

$$
\lim _{n \longrightarrow \infty} z_{n}=d\left(y_{n}, y_{n+1}\right)=r
$$

Suppose $r \geq 0$. Then,

$$
b^{s} \psi\left(d\left(y_{2 n+2}, y_{2 n+1}\right)\right) \leq b^{s} \alpha\left(d\left(y_{2 n+1}, y_{2 n}\right)\right) \beta\left(d\left(y_{2 n+1}, y_{2 n}\right)\right) .
$$

Taking the limit as $n \longrightarrow \infty$, we get $\psi(r) \leq \alpha(r) \leq \beta(r)$. Since $\alpha \in F$, by using (19), we have $\psi(r)<\alpha(r)<\beta(r)$ but this is a contradiction. Then, $r=0$. Hence,

$$
\lim _{n \longrightarrow \infty} z_{n}=d\left(y_{n}, y_{n+1}\right)=0
$$

Next, we have to show that $\left\{S x_{n}\right\}$ is a $b$-Cauchy sequence. We prove this by contradiction. Now, we suppose that $\left\{S x_{2 n}\right\}$ is not a $b$-Cauchy sequence. Then, for any $\varepsilon>0$, there exist two subsequences of positive integers $m_{k}$ and $n_{k}$ such that $n_{k}>m_{k}$ for all positive integers $k$,

$$
\begin{array}{r}
d\left(S x_{2 m_{k}}, S x_{2 n_{k}}\right)>\varepsilon, \\
d\left(S x_{2 m_{k}}, S x_{2 n_{k-2}}\right) \leq \varepsilon .
\end{array}
$$

From (34) and (35) and by using triangle inequality, we get

$$
\begin{aligned}
\varepsilon \leq & d\left(S x_{2 m_{k}}, S x_{2 n_{k-2}}\right) \leq d\left(S x_{2 m_{k}}, S x_{2 n_{k-2}}\right)+d\left(S x_{2 n_{k-2}}, S x_{2 n_{k-1}}\right) \\
& +d\left(S x_{2 n_{k-1}}, S x_{2 n_{k}}\right) .
\end{aligned}
$$

Let $k \longrightarrow \infty$ in the above inequality and by using (33), we obtain

$$
\lim _{k \longrightarrow \infty} d\left(S x_{2 m_{k}}, S x_{2 n_{k}}\right)=\varepsilon
$$

Again by using triangle inequality, we have

$$
d\left(S x_{2 n_{k}}, S x_{2 m_{k-1}}\right) \leq d\left(S x_{2 n_{k}}, S x_{2 m_{k}}\right)+d\left(S x_{2 m_{k}}, S x_{2 m_{k-1}}\right) .
$$

By taking limit as $k \longrightarrow \infty$ in above inequality and using (33)-(35), we get

$$
\lim _{k \longrightarrow \infty} d\left(S x_{2 n_{k}}, S x_{2 m_{k-1}}\right)=\varepsilon
$$

Moreover, we obtain

$$
\begin{aligned}
d\left(S x_{2 n_{k}}, S x_{2 m_{k}}\right) & \leq d\left(S x_{2 n_{k}}, S x_{2 n_{k+1}}\right)+d\left(S x_{2 n_{k+1}}, S x_{2 m_{k}}\right) \\
& =d\left(S x_{2 n_{k}}, S x_{2 n_{k+1}}\right)+d\left(P x_{2 n_{k}}, Q x_{2 m_{k-1}}\right) .
\end{aligned}
$$

Using inequalities (33)-(37) and letting $\lim _{k \longrightarrow \infty}$, we have

$$
\varepsilon \leq \lim _{k \longrightarrow \infty} d\left(P x_{2 n_{k}}, Q x_{2 m_{k-1}}\right)
$$

However, $\psi \in \Psi$, therefore

$$
\psi(\varepsilon) \leq \lim _{k \longrightarrow \infty} \psi\left(d\left(P x_{2 n_{k}}, Q x_{2 m_{k-1}}\right)\right) .
$$

From (18), we have

$b^{s} \psi\left(d\left(P x_{2 n_{k}}, Q x_{2 m_{k-1}}\right)\right) \leq b^{s} \alpha\left(d\left(S x_{2 n_{k}}, S x_{2 m_{k-1}}\right)\right) \beta\left(d\left(S x_{2 n_{k}}, S x_{2 m_{k-1}}\right)\right)$.

Taking limit as $k \longrightarrow \infty$ in the above inequality and using that fact that $\alpha \in F$, we have

$$
\lim _{k \longrightarrow \infty} \psi\left(d\left(P x_{2 n_{k}}, Q x_{2 m_{k-1}}\right)\right)<\beta(\varepsilon)
$$

From (43) and (44), and using (19), we obtain

$$
\psi(\varepsilon) \leq \lim _{k \longrightarrow \infty} \psi\left(d\left(P x_{2 n_{k}}, Q x_{2 m_{k-1}}\right)\right)<\beta(\varepsilon)<\psi(\varepsilon) .
$$

This is a contradiction. Therefore, $\left\{x_{2 n}\right\}$ is a $b$-Cauchy sequence, and hence, $\left\{x_{n}\right\}$ is a $b$-Cauchy sequence for all $n \in \mathbb{N}$. Hence, there exists $u \in X$ such that

$$
\lim _{n \rightarrow \infty} S x_{n}=u
$$

Next, claim that $u$ is a coincidence point of $P, Q$, and $S$. From (46) and the continuity of $S$, we get

$$
\lim _{n \longrightarrow \infty} S\left(S x_{n}\right)=S u
$$

From triangle inequality, we have

$$
\begin{aligned}
d(S u, P u) \leq & \left.d\left(S u, S\left(S x_{2 n+1}\right)\right)+d\left(S\left(T x_{2 n}\right)\right), P\left(S x_{2 n}\right)\right) \\
& +d\left(P\left(S x_{2 n}\right), T u\right)
\end{aligned}
$$

From (46) and (21), we have

$$
S x_{2 n} \longrightarrow u, P x_{2 n} \longrightarrow u \text {. }
$$


Since pair $(P, S)$ are compatible, then

$$
d\left(S\left(P x_{2 n}\right), P\left(S x_{2 n}\right)\right) \longrightarrow 0
$$

Using the continuity of $P$ and (58), we get

$$
d\left(P\left(S x_{2 n}\right), P u\right) \longrightarrow 0
$$

letting $k \longrightarrow \infty$ in (48) and using (47)-(50) together with (51), we find

$$
d(S u, P u) \leq 0
$$

which means that $S u=P u$. Similarly, from triangle inequality, we have

$$
\begin{aligned}
d(S u, Q u) \leq & \left.d\left(S u, S\left(S x_{2 n+2}\right)\right)+d\left(S\left(Q x_{2 n+1}\right)\right), Q\left(S x_{2 n+1}\right)\right) \\
& +d\left(Q\left(S x_{2 n+1}\right), Q u\right) .
\end{aligned}
$$

In a similar way, we obtain $d(S u, Q u) \leq 0$ which means that $S u=Q u$. Thus, we find that $S u=Q u=P u$, that is, $u$ is a coincidence point of $P, Q$, and $S$.

Now, we use the property (6) to show that $u$ is a common fixed point of $P, Q$, and $S$. For this, we prove that $P, Q$, and $S$ have a common fixed point. To prove this, we show that if $p$ and $q$ are coincidence points of $P, Q$, and $S$, i.e.,

$$
\begin{aligned}
& S p=P p=Q p, \\
& S q=P q=Q q .
\end{aligned}
$$

Then,

$$
S p=S q
$$

From our assumption mentioned in property (6), there exists $u_{0} \in X$ such that

$$
T p \preccurlyeq T u_{0}, T q \preccurlyeq T u_{0} .
$$

Now, we can define a sequence $\left\{S u_{n}\right\}$ as follows:

$$
S u_{2 n+1}=P u_{2 n}, S u_{2 n+2}=Q u_{2 n+1}, \forall n \in \mathbb{N}
$$

Again, we have

$$
P p=S p \preccurlyeq S u_{n}, P q=S q \preccurlyeq S u_{2 n}, \forall n \in \mathbb{N}
$$

Now, putting $x=u_{n}$ and $y=p$ in (18), we get

$$
\begin{aligned}
b^{s} \psi\left(d\left(S u_{2 n+1}, S p\right)\right) & =b^{s} \psi\left(d\left(P u_{2 n}, Q p\right)\right) \\
& \leq b^{s} \alpha\left(d\left(S u_{2 n}, S p\right)\right) \beta\left(d\left(S u_{2 n}, S p\right)\right)
\end{aligned}
$$

Since $\alpha \in F$, we have the next inequality:

$$
b^{s} \psi\left(d\left(S u_{2 n+1}, S p\right)\right) \leq b^{s} \beta\left(d\left(S u_{2 n}, S p\right)\right) .
$$

Similarly, again writing $y=u_{2 n}$ and $x=p$ in (18), we find

$$
b^{s} \psi\left(d\left(S u_{2 n+2}, S p\right)\right) \leq b^{s} \beta\left(d\left(S u_{2 n+1}, S p\right)\right) .
$$

By combining (60) and (61), we obtain for all $n \in \mathbb{N}$,

$$
b^{s} \psi\left(d\left(S u_{2 n+1}, S p\right)\right) \leq b^{s} \beta\left(d\left(S u_{2 n}, S p\right)\right) .
$$

Consequently, by using property of $\psi(t)$ and $\beta(t)$, we get

$$
d\left(S u_{2 n+1}, S p\right) \leq d\left(S u_{2 n}, S p\right) .
$$

Therefore, there exists $r \geq 0$ such that

$$
\lim _{n \rightarrow \infty} d\left(S u_{n}, S p\right)=r
$$

Let $r>0$, then from (18), we obtain

$$
b^{s} \psi\left(d\left(S u_{2 n+1}, S p\right)\right) \leq b^{s} \alpha\left(\left(d\left(S u_{2 n}, S p\right)\right) \beta\left(d\left(S u_{2 n}, S p\right)\right)\right.
$$

on taking limit as $n \longrightarrow \infty$ and using (19), we get

$$
\psi(r)<\beta(r)<\psi(r)
$$

This is a contradiction. Thus, $r=0$ and from (64), we obtain

$$
\lim _{n \rightarrow \infty} d\left(S u_{n}, S p\right)=0
$$

In the same pattern, we can show that

$$
\lim _{n \rightarrow \infty} d\left(S u_{n}, S q\right)=0
$$

Now, using the fact that the limit is unique and by using (55)-(68), we can write

$$
\begin{aligned}
\lim _{n \longrightarrow \infty} d\left(S\left(P u_{2 n}, P\left(S u_{2 n}\right)\right)\right) & =0, \lim _{n \longrightarrow \infty} d\left(S\left(Q u_{2 n+1}, Q\left(S u_{2 n}+1\right)\right)=0,\right. \\
\lim _{n \longrightarrow \infty} P u_{2 n} & =S p=S q, \\
\lim _{n \longrightarrow \infty} Q u_{2 n+1} & =S p=S q .
\end{aligned}
$$

Since the pair $P, S$ and $Q, S$ are compatible, we have $\lim _{n \longrightarrow \infty} d\left(S\left(P u_{2 n}, P\left(S u_{2 n}\right)\right)\right)=0, \lim _{n \longrightarrow \infty} d\left(S\left(Q u_{2 n+1}, Q\left(S u_{2 n}+1\right)\right)=0\right.$.

Let us take $z=S p$. Consider

$$
d(S z, P z) \leq d\left(S z, S\left(P u_{2 n}\right)\right)+d\left(S\left(T u_{2 n}\right), P\left(S u_{2 n}\right)\right)+d\left(P\left(S u_{2 n}\right), P z\right) .
$$

Letting $n \longrightarrow \infty$ and using the continuity of $P$, we get the above inequality as

$$
d(S z, P z) \leq 0 \text {. }
$$


That is, $S z=P z$ and $z$ is coincidence point of $P$ and $S$. Similarly, $d(S z, Q z) \leq 0$. That is, $R z=S z$ and $z$ is the coincidence point of $Q$ and $S$. Hence, from (55), we have

$$
z=S p=S z=P z=Q z
$$

This proves that $z$ is a common fixed point of $P, Q$, and $S$. Now, we show that $z$ is unique common fixed point. We will show this by contradiction. Assume that $z$ is not unique, therefore, there exists another fixed point $v$ as

$$
v=S p=S v=P v=Q v .
$$

By using (55), we have $S v=S z$. Hence, we get

$$
v=S v=S z=z
$$

but this is a contradiction to our assumption, and hence, a common fixed point is unique.

An example is given to support the main result because there is limited examples in the literature.

Example 2. Let $X=\mathbb{R}$ is the set of real numbers and a $b$-metric $d: X \times X \longrightarrow 0, \infty$ is defined as

$$
d(x, y)=(x-y)^{2}, \forall x, y \in X
$$

with coefficient $s=2$ and $b=0.01$. Consider three selfmaps $P, Q, S: \mathbb{R} \longrightarrow \mathbb{R}$ defined as

$$
P x=2 x, Q x=5-2 x, S x=\frac{x}{3} .
$$

Define maps $\psi, \beta:[0, \infty) \longrightarrow[0, \infty)$ and $\alpha:[0, \infty) \longrightarrow$ $[0,1]$ as

$$
\begin{aligned}
\psi(t) & =\frac{t}{2}, \\
\beta & =\frac{t}{3}, \\
\alpha(t) & =\frac{7}{3} .
\end{aligned}
$$

After substituting values in (18),

$$
b^{s} \psi(d(P x, Q y)) \leq \alpha((d(S x, S y)) \beta(d(S x, S y)),
$$

we have

$$
\begin{aligned}
& 0.01^{2}\left(\psi(2 x-5+2 y)^{2}\right) \leq \alpha\left(d\left(\frac{x}{3}, \frac{y}{3}\right)\right) \beta\left(d\left(\frac{x}{3}, \frac{y}{3}\right)\right), \\
& 0.01^{2}\left(\frac{(2 x-5+2 y)^{2}}{2}\right) \leq \frac{7}{3}\left(\frac{\left.(x-y)^{2}\right)}{27}\right) .
\end{aligned}
$$

Now, for $(x, y)=(0,1)$, we get

$$
0.00045 \leq 0.08
$$

For $(x, y)=(1,2)$, we have

$$
0.00005 \leq 0.08
$$

Then, clearly, three maps $P, Q$, and $S$ are generalized $(\psi, \alpha, \beta)$-weak contraction for all values of $x, y \in \mathbb{R}$.

\section{An Application to Fredholm Integral Equations}

In this section, applying Theorem 17, we give an existence theorem for common solutions of Fredholm integral equations where the upper limits of equations are taken to be the coefficient of $b$-metric space $s \geq 1$. Here, we consider the following integral equations:

$$
\int_{0}^{s} P_{1}(t, l, v(l)) d l+\varkappa(t)
$$

$$
\int_{0}^{s} P_{2}(t, l, v(l)) d l+\varkappa(t)
$$

for all $t \in[0, s]$ where $s \geq 1$. Let us consider the space $X=C[I]$ $(I=[0, s])$ is a set of continuous functions defined on $[0, s]$. Obviously, the space $C[I]$ with the metric $d(p, q)=\max _{t \in I} \mid x(t)$ $-y(t) \mid$ for all $x, y \in X$ is a complete metric space.

Here, $b$-metric space is defined on the partially ordered set. So, $X$ can be prepared with partial order $\preccurlyeq$ given by

$$
\text { for all } x, y \in X, x \preccurlyeq y: \Longleftrightarrow x(t) \preccurlyeq y(t) \text {, for all } t \in I \text {. }
$$

Theorem 18. Suppose the following hypotheses hold:

(1) $P_{1}, P_{2}: I \times I \times R \longrightarrow R \quad$ and $\quad \lambda: R \longrightarrow R \quad$ are
continuous

(2) The following inequalities hold:

$$
\begin{aligned}
& P_{1}(t, l, u(l)) \leq P_{2}\left(t, l, \int_{0}^{s} P_{1}(l, r, u(r)) d r+\varkappa(l),\right. \\
& P_{2}(t, l, u(l)) \leq P_{1}\left(t, l, \int_{0}^{s} P_{2}(l, r, u(r)) d r+\varkappa(l)\right.
\end{aligned}
$$

(3) There exists a continuous function $\xi: I \times I \longrightarrow R_{+}$ such that

$b^{s}\left|P_{1}(t, l, x)-P_{2}(t, l, y)\right| \leq \xi(t, l) \sqrt{\frac{\log \left[(x-y)^{2}+s\right]}{x-y}}$, 
for all $t, l \in I$ and $x, y \in X$ such that $x \leqslant y$.

$$
\max _{t \in I} \xi(t, i) \int_{0}^{s} \xi(t, i)^{2} d l \leq \frac{1}{s}
$$

Then, the integral equations (83) and (84) have the unique solution $v^{*} \in C(I)$.

Proof. Let us define $P, Q: C(I) \longrightarrow C(I)$ by

$$
\begin{aligned}
& P_{x}(t)=\int_{0}^{s} T_{1}(t, l, v(l)) d l+\varkappa(t), \\
& Q_{x}(t)=\int_{0}^{s} T_{2}(t, l, v(l)) d l+\varkappa(t),
\end{aligned}
$$

where $t \in I$ and $p \in C(I)$. Here, $P$ and $Q$ are considered to be weakly increasing function according to the requirement of our result.

Now, for all $p, q \in C(I)$ such that $p \preccurlyeq q$, we have

$$
\begin{aligned}
b^{s}\left|P x(t)-Q_{y}(t)\right| & =\int_{0}^{s}\left|P_{1}(t, l, v(l))-P_{2}(t, l, v(l))\right| d l \\
& \leq \int_{0}^{s} \xi(t, l) \sqrt{\frac{\log \left[(x(l)-y(l))^{2}+s\right]}{x(l)-y(l)}} d l,
\end{aligned}
$$

where $b \in(0,1)$.

Using the Cauchy-Schwarz inequality in the R.H.S., we get

$$
\begin{aligned}
& \int_{0}^{s} \xi(t, l) \sqrt{\frac{\log \left[(x(l)-y(l))^{2}+s\right]}{x(l)-y(l)}} d l \\
& \quad \leq\left(\sqrt{\int_{0}^{s} \xi^{2}(t, l) d l}\right)\left(\sqrt{\int_{0}^{s} \frac{\log \left[(x(l)-y(l))^{2}+s\right]}{x(l)-y(l)}} d l\right) .
\end{aligned}
$$

By using hypotheses (4), we have

$$
\begin{aligned}
& \int_{0}^{s} \xi(t, l) \sqrt{\frac{\log \left[(x(l)-y(l))^{2}+s\right]}{x(l)-y(l)} d l} \\
& \quad \leq \sqrt{\frac{1}{s} \sqrt{\int_{0}^{s} \frac{\log \left[(x(l)-y(l))^{2}+s\right]}{x(l)-y(l)}} d l} \\
& \quad \leq\left(\sqrt{\left.\frac{1}{s}\right)\left(\sqrt{\frac{\log \left[(d(x, y))^{2}+s\right]}{d(x, y)}}\right)(\sqrt{s})}\right. \\
& \quad \leq \sqrt{\frac{\log \left[(d(x, y))^{2}+s\right]}{d(x, y)} .}
\end{aligned}
$$

This implies the following:

$$
\begin{aligned}
b^{s}\left|P_{x}(t)-Q_{y}(t)\right|= & b^{s} d\left(P_{x}, P_{y}\right) \\
\leq & \sqrt{\frac{\log \left[(d(x, y))^{2}+s\right]}{d(x, y)}}, b^{s} d\left(P_{x}, P_{y}\right)^{2} \\
\leq & \frac{\log \left[(d(x, y))^{2}+s\right]}{d(x, y)} \\
\leq & \left(\sqrt{\frac{\log \left[(d(x, y))^{2}+s\right]}{d(x, y)}}\right) \\
& \cdot\left(\sqrt{\log \left[(d(x, y))^{2}+s\right]}\right),
\end{aligned}
$$

Suppose we choose the values of $\alpha, \beta, \psi$ as $\sqrt{\left(\log \left[t^{2}+s\right] / t\right)}, \sqrt{\log \left[t^{2}+s\right.}$, and $t^{2}$, respectively. Therefore, from inequality (93),

$$
b^{s} \psi(d(P x, Q y)) \leq \alpha((d(S x, S y)) \beta(d(S x, S y)), \forall x \geq y
$$

Since all the hypotheses of Theorem 17 are satisfied so there exists a unique common fixed point $v^{*} \in C(I)$ of $P$ and $Q$, that is, the solution of equations (83) and (84).

\section{Data Availability}

No data were used to support this study.

\section{Conflicts of Interest}

The authors declare that they have no competing interests.

\section{Authors' Contributions}

The authors equally conceived the study, participated in its design and coordination, drafted the manuscript, participated in the sequence alignment, and read and approved the final manuscript.

\section{Acknowledgments}

The authors thank the Basque Government for its support of this work through Grant IT1207-19. This study is supported by Ege University Scientific Research Projects Coordination Unit (Project Number FGA-2020-22080). The second author is thankful to Higher Education Commission of Pakistan (HEC).

\section{References}

[1] P. Borisut, P. Kumam, V. Gupta, and N. Mani, "Generalized $(\psi, \alpha, \beta)$-weak contractions for initial value problems," Mathematics, vol. 7, no. 3, p. 266, 2019.

[2] S. Czerwik, "Contraction mappings in b-metric spaces," Acta mathematica et informatica universitatis ostraviensis, vol. 1, pp. 5-11, 1993.

[3] N. Bourbaki, Topologie Generale, Herman, Paris, 1974. 
[4] I. A. Bakhtin, "The contraction mapping principle in almost metric spaces," Functional Analysis, Gosudarstvennogo Pedagogichoskogo Instituta Unianowsk, vol. 30, pp. 26-37, 1989.

[5] M. A. Khamsi and N. Hussain, "KKM mappings in metric type spaces," Nonlinear Analysis, vol. 73, no. 9, pp. 3123-3129, 2010.

[6] H. Alsamir, M. Salmi, M. D. Noorani, W. Shatanawi, and F. Shaddad, "Generalized Berinde-type $(\eta, \xi, v, \theta)$-contractive mappings in b-metric spaces with an application," Journal of Mathematical Analysis, vol. 7, no. 6, pp. 1-12, 2016.

[7] A. H. Ansari, O. Ege, and S. Radenovic, "Some fixed point results on complex valued $\mathrm{G}_{\mathrm{b}}$-metric spaces," Revista de la Real Academia de Ciencias Exactas, Físicas y Naturales. Serie A. Matemáticas, vol. 112, no. 2, pp. 463-472, 2018.

[8] H. Aydi, M.-F. Bota, E. Karapinar, and S. Mitrovic, "A fixed point theorem for set-valued quasi-contractions in b-metric spaces," Fixed Point Theory and Applications, vol. 88, 8 pages, 2012.

[9] P. Baradol, D. Gopal, and S. Radenovic, "Computational fixed points in graphical rectangular metric spaces with application," Journal of Computational and Applied Mathematics, vol. 375 , p. 112805, 2020.

[10] L. Budhia, H. Aydi, A. H. Ansari, and D. Gopal, "Some new fixed point results in rectangular metric spaces with an application to fractional-order functional differential equations," Nonlinear Analysis, vol. 25, no. 4, pp. 580-597, 2020.

[11] O. Ege, "Complex valued rectangular b-metric spaces and an application to linear equations," Journal of Nonlinear Sciences and Applications, vol. 8, no. 6, pp. 1014-1021, 2015.

[12] O. Ege, "Complex valued $\mathrm{G}_{\mathrm{b}}$-metric spaces," Journal of Computational Analysis and Applications, vol. 21, no. 2, pp. 363-368, 2016.

[13] O. Ege, "Some fixed point theorems in complex valued $G_{b}$ -metric spaces," Journal of Nonlinear and Convex Analysis, vol. 18, no. 11, pp. 1997-2005, 2017.

[14] O. Ege, C. Park, and A. H. Ansari, "A different approach to complex valued $\mathrm{G}_{\mathrm{b}}$-metric spaces," Advances in Difference Equations, vol. 2020, no. 152, 13 pages, 2020.

[15] A. Gholidahneh, S. Sedghi, O. Ege, Z. D. Mitrovic, and M. de la Sen, "The Meir-Keeler type contractions in extended modular b-metric spaces with an application," AIMS Mathematics, vol. 6, no. 2, pp. 1781-1799, 2021.

[16] D. Gopal, M. Abbas, D. K. Patel, and C. Vetro, "Fixed points of $\alpha$-type F-contractive mappings with an application to nonlinear fractional differential equation," Acta Mathematica Scientia, vol. 36, no. 3, pp. 957-970, 2016.

[17] N. Hussain, V. Parvaneh, J. R. Roshan, and Z. Kadelburg, "Fixed points of cycle weakly $(\psi, \varphi, \mathrm{L}, \mathrm{A}, \mathrm{B})$-contractive mappings in ordered b-metric spaces with applications," Fixed Point Theory and Applications, vol. 2013, 256 pages, 2013.

[18] M. Iqbal, A. Batool, O. Ege, and M. de la Sen, "Fixed point of almost contraction in b-metric spaces," Journal of Mathematics, vol. 2020, Article ID 3218134, 6 pages, 2020.

[19] H. Lakzian, D. Gopal, and W. Sintunavarat, "New fixed point results for mappings of contractive type with an application to nonlinear fractional differential equations," Journal of Fixed Point Theory and Applications, vol. 18, no. 2, pp. 251-266, 2016.

[20] A. R. Lucas, "A fixed point theorem for a general epidemic model," Journal of Mathematical Analysis and Applications, vol. 404, no. 1, pp. 135-149, 2013.
[21] B. Samet, C. Vetro, and P. Vetro, "Fixed point theorems for -contractive type mappings," Nonlinear Analysis, vol. 75, no. 4, pp. 2154-2165, 2012.

[22] T. Suzuki, "Basic inequality on a b-metric space and its applications," Journal of inequalities and applications, vol. 2017, no. $1,2017$.

[23] T. Kamran, M. Samreen, and Q. U. L. Ain, "A generalization of b-metric space and some fixed point theorems," Mathematics, vol. 5, no. 2, p. 19, 2017.

[24] Y. I. Alber and S. Guerre-Delabriere, "Principle of weakly contractive maps in Hilbert spaces," in New Results in Operator Theory and Its Applications, I. Gohberg and Y. Lyubich, Eds., vol. 98, pp. 7-22, Operator Theory: Advances and Applications, Birkhäuser, Basel, Switzerland, 1997.

[25] P. N. Dutta and B. S. Choudhury, "A generalisation of contraction principle in metric spaces," Fixed Point Theory and Applications, vol. 2008, no. 1, 2008.

[26] B. E. Rhoades, "Some theorems on weakly contractive maps," Nonlinear Analysis, vol. 47, no. 4, pp. 2683-2693, 2001.

[27] Q. Zhang and Y. Song, "Fixed point theory for generalized $\varphi$ -weak contractions," Applied Mathematics Letters, vol. 22, no. 1, pp. 75-78, 2009.

[28] D. Doric, "Common fixed point for generalized $(\psi, \varphi)$-weak contractions," Applied Mathematics Letters, vol. 22, no. 12, pp. 1896-1900, 2009.

[29] I. Altun and H. Simsek, "Some fixed point theorems on ordered metric spaces and application," Fixed Point Theory and Applications, vol. 2010, no. 1, 2010.

[30] G. Jungck, "Compatible mappings and common fixed points," International Journal of Mathematics and Mathematical Sciences, vol. 9, no. 4, 779 pages, 1986. 